\title{
Supporting Content
}

Asymmetric Light Absorption and Reflection in Freestanding Nanostructured Membranes

Serkan Butun and Koray Aydin*

Comparison of absorption with respect to perforated silver film without any dielectric and $\underline{\text { blank silver film }}$

In figure S1, we present a comparison of simulated absorption spectra of front and back illuminated $\mathrm{Ag} / \mathrm{Si}_{3} \mathrm{~N}_{4}$ double layer structure, perforated $\mathrm{Ag}$ layer without and dielectric and a blank Ag film. The thickness of the Ag film was kept at $50 \mathrm{~nm}$ in all calculations. For perforation we used digitized SEM images of structures with periodicity $350 \mathrm{~nm}$.

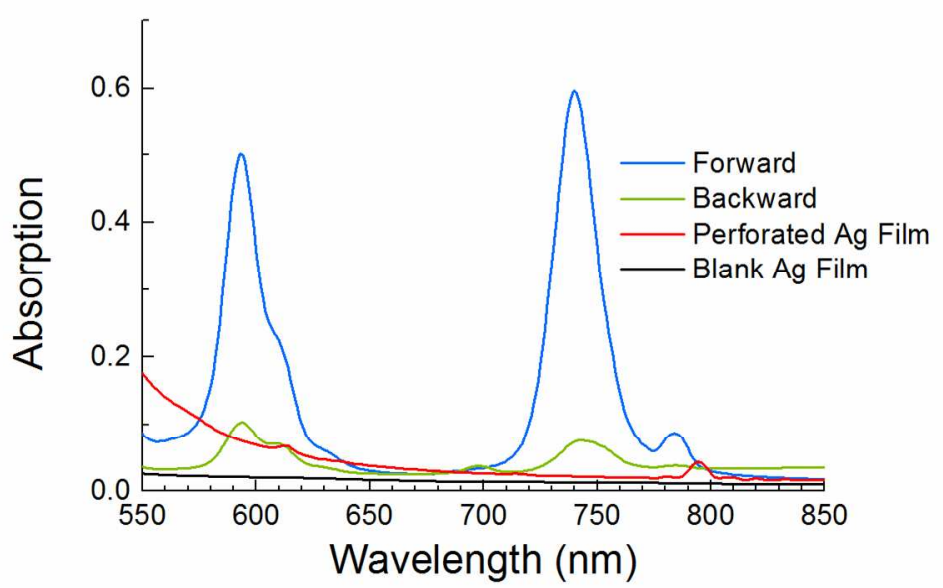

Figure S1: The comparison of spectral absorption of $\mathrm{Ag} / \mathrm{Si}_{3} \mathrm{~N}_{4}$ heterostructure with hypothetical Ag films with and without perforations.

\section{Additional cross-sectional absorption maps:}

The maximum absorption occurs at $\mathrm{Ag} / \mathrm{Si}_{3} \mathrm{~N}_{4}$ interface. For clarity, cross-sectional absorption maps at vertical planes are represented in Figure S2. The absorption only occurs in Ag. 


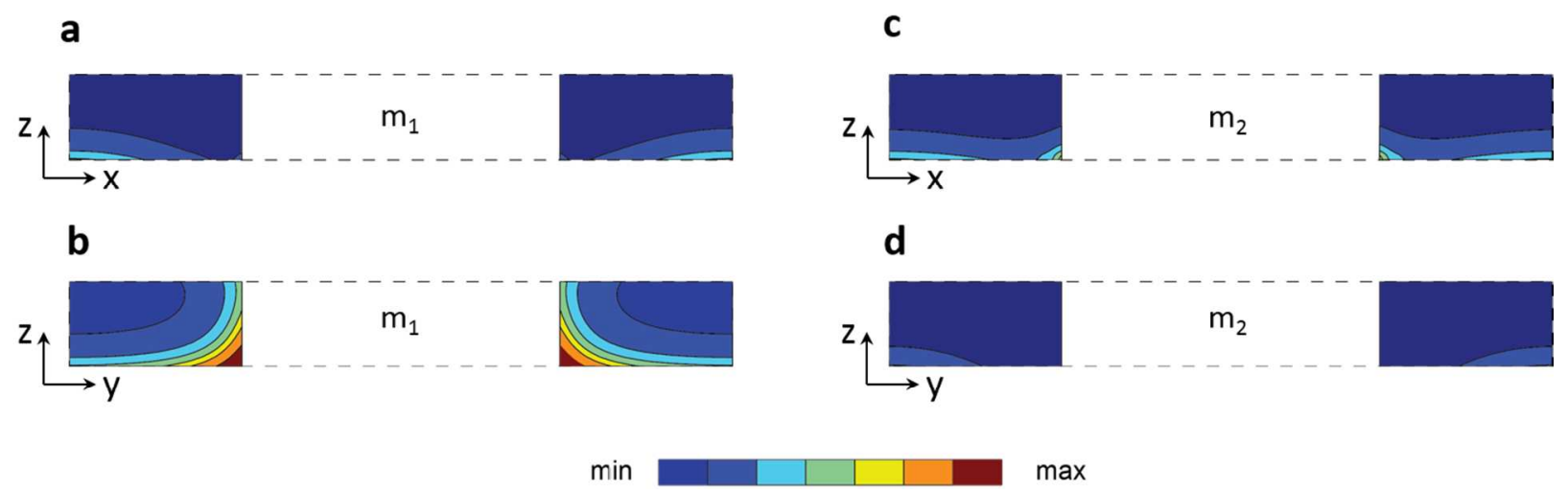

Figure S2: Calculated cross-sectional absorption profiles in the unit cell of the metasurface absorber. (a, b) mode $\mathrm{m}_{1}$ at $x z$ - and $y z$ plane, respectively. (c, d) mode $\mathrm{m}_{2}$ at $x z$ - and $y z$ plane, respectively. The colorbar applies to all. The plane wave illumination along $-z$-direction (front illumination) with E-field along $y$-direction is used in all cases.

\section{Surface current density:}

Figure $\mathrm{S} 3$ represents the calculated surface current density at $\mathrm{Ag} / \mathrm{Si}_{3} \mathrm{~N}_{4}$ interface for both modes.

The electrical character of both resonances is clearly illustrated.

a

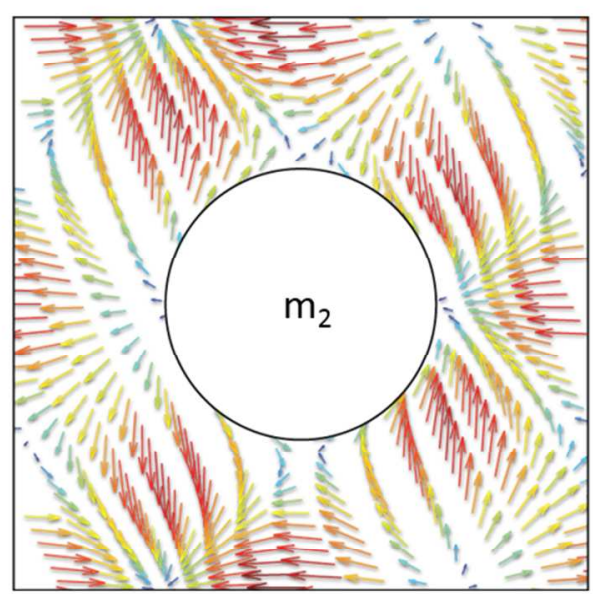

b

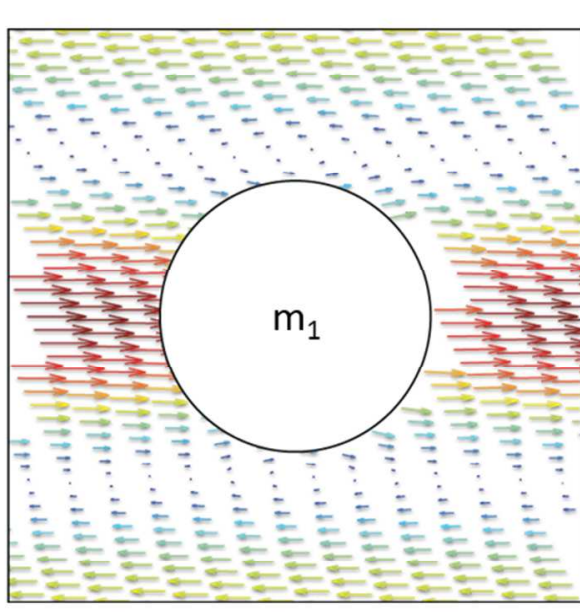

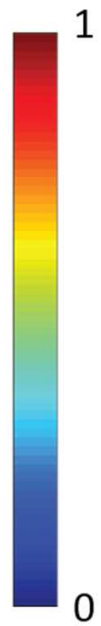

Figure S3: Surface current density at the $\mathrm{Ag} / \mathrm{Si}_{3} \mathrm{~N}_{4}$ interface for (a) mode $\mathrm{m}_{2}$ and (b) mode $\mathrm{m}_{1}$. 